\title{
Serological study of viral hepatitis
}

\author{
M. Raghavendra Rao', Rashmi P. Mahale,", Sowmya G.S ${ }^{3}$, Ranjijha Shankare Gowda ${ }^{4}$ \\ ${ }^{1-3}$ Associate Professor, ${ }^{4}$ Assistant Professor Dept. of Microbiology, JSS Academy of Higher Education and Research Mysore \\ Karnataka, India
}

*Corresponding Author:

Email: rashmimahale@gmail.com

\begin{abstract}
Introduction: Viral hepatitis is caused by hepatotropic virus like hepatitis A, B, C, D, E and G. Viral hepatitis is a major public health problem in India, which is hyperendemic for HAV and HEV. The present study titled 'serological study of viral hepatitis' is undertaken to detect the rate of different hepatitis viral infections, also to identify coexistence of any of these viral hepatitis and an attempt is also made to detect the presence of other hepatitis B virus markers which would help in understanding the prognosis of hepatitis.

Material and Methods: Serum samples were tested for detection of hepatitis A IgM, hepatitis B surface antigen, hepatitis C antibody and hepatitis E IgM antibody using specific ELISA kits.

For patients showing positive results to HBsAg tests further markers like anti-HBs, HBeAg and anti-HBc were detected by ELISA.

Results: A total of 2760 samples were screened for the presence of HAV, HBV, HCV and HEV. And seropositivity was detected. 67 cases were positive for HBsAg (2.7\%), 12 for hepatitis A (35.5\%), 2 each were positive for hepatitis C (0.69\%) and hepatitis E (17\%) respectively. Of 2760 patients tested, 2 co-infections occurred HAV along with HBV and HAV with HEV.

Conclusion: In this study a total of 2760 cases were screened for viral hepatitis infections. The patients showed positivity to HAV, HBsAg, HCV, HEV and some of the HBV markers. And some co infections were also seen. It is important to diagnose viral hepatitis to limit the spread of this contagious disease. This is particularly important in the routine testing of potential blood donors.
\end{abstract}

Keywords: Hepatitis, ELISA, Seroprevalence, Diagnosis.

\section{Introduction}

Viral hepatitis is a major public health problem throughout the world affecting several hundreds of millions of people. Hepaititis literally means inflammation of the liver. This disease can range from being subclinical to life threatening. Viruses are the most common and significant causes of hepatitis ${ }^{1}$ Viral hepatitis most commonly caused by one of the six hepatotrophic viruses: hepatitis A, B, C, D, E, and G virus (HAV to HEV and $\mathrm{HGV}$ ). These agents differ in their biological, immunological, pathological and epidemiological characteristics.

The infectious nature of hepatitis was long unrecognized because the disease tends to occur sporadically and because jaundice, a prominent clinical sign, has many diverse causes. Since the time of Virchow the disease was believed to result from obstruction of the common bile duct by a plug of mucus, and it was known as acute catarrhal jaundice. In 1942 voeght first transmitted hepatitis by feeding a patient's duodenal contents to volunteers. Subsequently, it was found that the etiologic agents are filterable and that the disease may be transmitted in two ways; by the intestinal-oral route (infectious hepatitis) or by the injection of infected blood or its product (serum hepatitis). However, the difference in transmission are not absolute, since the virus of so called infectious hepatitis can also produce diseases when inoculated parentally, and experimentally the virus of serum hepatitis has been transmitted orally. ${ }^{3}$
Viral hepatitis is a major public health problem in India, which is hyperendemic for HAV and HEV. Seroprevalence studies reveal that $90 \%-100 \%$ of the population acquires anti-HAV antibody and becomes immune by adolescence. Many epidemics of HEV have been reported from India. HAV related liver disease is uncommon in India and occurs mainly in children. $\mathrm{HEV}$ is also the major cause of sporadic adult acute viral hepatitis and acute liver failure (ALF). Pregnant women and patients with chronic liver disease (CLD) constitute the high risk groups to contract HEV infection, and HEV-induced mortality among them is substantial, which underlines the need for preventive measures for such groups. Children with HAV and HEV coinfection are prone to develop ALF. ${ }^{4}$

India has intermediate HBV endemicity, with a carrier frequency of $2 \%-4 \%$. HBV is the major cause of CLD and hepatocellular carcinoma (HCC). Chronic HBV infection in India is acquired in childhood, presumably before 5 years of age, through horizontal transmission. Vertical transmission of HBV in India is considered to be infrequent. Inclusion of $\mathrm{HBV}$ vaccination in the expanded programme of immunization is essential to reduce the HBV carrier frequency and disease burden. HBV genotypes A and D are prevalent in India, which are similar to the HBV genotypes in the West. ${ }^{4}$

$\mathrm{HCV}$ infection in India has a population prevalence of around $1 \%$, and occurs predominantly through 
transfusion and the use of unsterile glass syringes. HCV genotypes 3 and 2 are prevalent in $60 \%-80 \%$ of the population and they respond well to a combination of interferon and ribavirin. About $10 \%-15 \%$ of CLD and $\mathrm{HCC}$ are associated with $\mathrm{HCV}$ infection in India. $\mathrm{HCV}$ infection is also a major cause of post-transfusion hepatitis. $^{4}$

HDV infection is infrequent in India and is present about $5 \%-10 \%$ of patients with HBV-related liver disease. HCC appears to be less common in India than would be expected from the prevalence rates of $\mathrm{HBV}$ and HCV. The high disease burden of viral hepatitis and related CLD in India, calls for the setting up of a hepatitis registry and formulation of governmentsupported prevention and control strategies. ${ }^{4}$

The present study titled 'serological study of viral hepatitis' is undertaken to detect the rate of different hepatitis viral infections, also to identify coexistence of any of these viral hepatitis and an attempt is also made to detect the presence of other hepatitis B virus markers which would help in understanding the prognosis of hepatitis.

\section{Material and Methods}

Serum samples received in the laboratory from clinically suspected hepatitis cases and preoperative screening for hepatitis was the material. These samples were tested for detection of hepatitis A IgM, hepatitis B surface antigen, hepatitis $\mathrm{C}$ antibody and hepatitis E IgM antibody using specific ELISA kits.

For patients showing positive results to $\mathrm{HBsAg}$ tests further markers like anti-HBs, $\mathrm{HBeAg}$ and anti$\mathrm{HBc}$ were detected by ELISA.

\section{Results}

In the present study 2760 serum samples were screened for detection of different viral hepatitis agents.1640 were males and 1130 were females and maximum number screened were between the age group of 31 to 50 years. Of the total samples, highest numbers of samples received were screened for Hepatitis B (2429) infection, followed by Hepatitis C (287), Hepatitis A (32) and Hepatitis E (12). The samples obtained differed in age and sex and are shown in Table 1.

Table 1: Record of total cases tested

\begin{tabular}{|c|c|c|c|c|c|c|c|c|c|}
\hline Test done & Age group & \multicolumn{2}{|c|}{ HAV } & \multicolumn{2}{c|}{ HBV } & \multicolumn{2}{c|}{ HCV } & \multicolumn{2}{c|}{ HEV } \\
\hline & & Male & Female & M & F & M & F & M & F \\
\hline \multirow{5}{*}{ ELISA } & $0-10$ & 5 & 4 & 69 & 26 & 2 & 3 & 2 & 0 \\
\cline { 2 - 11 } & $11-20$ & 3 & 3 & 113 & 125 & 9 & 8 & 0 & 2 \\
\cline { 2 - 11 } & $21-30$ & 3 & 1 & 277 & 345 & 22 & 14 & 0 & 0 \\
\cline { 2 - 11 } & $31-40$ & 3 & 1 & 251 & 163 & 26 & 18 & 2 & 0 \\
\cline { 2 - 11 } & $41-50$ & 2 & 1 & 241 & 151 & 41 & 19 & 2 & 0 \\
\cline { 2 - 11 } & $51-60$ & 2 & 0 & 201 & 100 & 40 & 14 & 2 & 0 \\
\cline { 2 - 10 } & $61-70$ & 2 & 0 & 186 & 60 & 32 & 13 & 1 & 0 \\
\cline { 2 - 10 } & $71 \&$ above & 2 & 0 & 77 & 44 & 41 & 5 & 1 & 0 \\
\hline Total & & 22 & 10 & 1415 & 1014 & 193 & 94 & 10 & 2 \\
\hline
\end{tabular}

67 cases were positive for HBsAg (2.7\%), 12 for hepatitis A $(35.5 \%), 2$ each were positive for hepatitis $\mathrm{C}(0.69 \%)$ and hepatitis $\mathrm{E}(17 \%)$ respectively. One HBsAg sample was positive for Anti-HBsAg out of 67 HBsAg positives and 13 were found to have $\mathrm{HBc}$ IgM and one with $\mathrm{HBeAg}$ positivity out of 28 tested. The detection of Anti-HBe out of the 67 positive $\mathrm{HBsAg}$ was found to be negative. Of 2760 patients tested, 2 coinfections occurred HAV along with HBV and HAV with $\mathrm{HEV}$.

\section{Discussion}

The main objective of the study was to detect the distribution of viral hepatitis cases and to detect any co- existing infections in the study. The total no of serum samples received from suspected cases of viral hepatitis for detection of respective antigen/ antibody were 2760 , of which $83(3 \%)$ samples were found to be positive for $\mathrm{HAV}, \mathrm{HBsAg}, \mathrm{HCV}$ and HEV. Of the 2760 samples; 2429 were tested for HBsAg of which 67 (2.7\%) were positive, 32 were tested for HAV of which $12(37.5 \%)$ were positive, 287 were tested for $\mathrm{HCV}$ of which 2 $(0.69 \%)$ were positive and 12 were tested for HEV of which $2(17 \%)$ were positive. The rate of serologically proved viral hepatitis was 3\% (83 out of 2760 samples tested).In a study at Australia a very high rate of viral hepatitis has been reported(66/82).This high rate is probably due to specific selection of clinically \& 
Biochemically proven cases of Viral hepatitis. Similarly another study from Washington also has reported a very high rate of viral hepatitis seropositivity. But in the present study majority of samples received were for pre operative screening and clinically suspected cases of viral hepatitis were only 44 . Going by this data in the study overall rate of proved viral hepatitis is about $33 \%$. 5,6

Out of the 2760 samples 2429 serum samples were screened for detection of hepatitis B infection. 67 of these were HBsAg positive (2.7\%). This is the rate of Hepatitis B viral seropositivity worldwide, though some researchers have quoted higher rate of positivity for Hepatitis B infection. Hepatitis B infection mainly occurs by transfusion of contaminated blood \& blood products. Only about $15 \%$ of infected individuals show symptoms of acute hepatitis. Hence the low rate of seropositivity. The data may only be the tip of an Ice berg, a detailed population based study may reveal an higher rate of Hepatitis B infection. In India most of these cases \& also Hepatitis $\mathrm{C}$ viral infected individuals are detected accidentally like at the time of blood donation, preoperative screening, posted for dialysis, etc. Further screening of the positive individuals will help to learn to prognosis of hepatitis B positive cases.

The study detected 12/37 cases of Hepatitis A seropositivity $(37.5 \%)$. Hepatitis A viral infection is one of the commonest causes of Hepatitis in developing countries where there is poor public health awareness. It is always transmitted by contaminated food and water. It is commonly referred to as Infective Hepatitis / short incubation Hepatitis. Fortunately complications are minimal $\&$ it subsides spontaneously. One infection (either subclinical or symptomatic) induces a lifelong immunity. But for this fact the rate of Hepatitis A infection would have been much higher than the observed data. Similar rate of Hepatitis A seropositivity has been observed by many workers all over the world both in developed \& developing contries. ${ }^{5-8}$

Hepatitis E virus is another enterically transmitted viral agent causing acute viral hepatitis in many places. This virus is being increasingly detected during the recent times in all cases of viral hepatitis which are seronegative for Hepatitis A virus. This virus along with Hepatitis $C$ virus was earlier categorized broadly as Non A Non B hepatitis. With the development of a sensitive and specific ELISA kit the HEV cases are being more frequently detected in many countries.

In the present study HEV seropositivity was $2 / 12$ $(17 \%)$.similar observation (18.8\%) is made in a 3 year study by Mishra et $\mathrm{al}^{9}$ and also by Chau et $\mathrm{al}^{7}(11.5 \%)$ who ha broadly categorized all as non A Non B hepatitis. In recent studies it has been found that in pregnancy Hepatitis E viral infection is more common and also complication including fatality is observed in pregnant women. The exact reason for this fatal outcome is yet to be analyzed. During the coming years more cases of Hepatitis E infection may be increasingly detected world wide. ${ }^{8.10}$

Out of 287 samples tested for Hepatitis C viral seropositivity were positive for Hepatitis C Antibody $(0.7 \%)$. Data of the previous years showed an higher rate of Hepatitis $\mathrm{C}$ seropositivity, more commonly in renal dialysis patients (4\%). Implementation of very strict guidelines for screening blood donors, organ donors and dialysis patients the rate of Hepatitis $\mathrm{C}$ viral infection is gradually declining. Hepatitis $\mathrm{C}$ viral hepatitis also is detected very late in the course of disease development. Only 15\% show manifestation during acute phase. But chronic infection by this virus is more serious and may progress to hepatocellular carcinoma or cirrhosis of liver than other viruses. It is more severe in HIV and immunocompromised patients. ${ }^{11}$

Co-existence of 2 or more viral agents is often detected in some of the patients. This scenario causes confusion in the management of such cases. There are reports of coexisting Hep B \& Hep C viral infections. Similarly co existence of Hepatitis A \& E infections also is not unusual. The present aimed to detect such coexistence among the patients. The combination of co existence is mainly based on route of transmission. Surprisingly in the study we could detect one case of Hepatitis A infection coexisting with Hepatitis B infection. This finding can be explained by the hypothesis that the patient had previous Hepatitis B infection which did not manifest as Hepatitis and was not detected; this patient now developed symptoms of viral hepatitis of short incubation. Thus it can be deduced that Hepatitis A viral infection was secondary to hepatitis B infection.

One case of Hepatitis A coexisting with Hepatitis E infection was detected in the study. This can be a natural phenomenon as both are transmitted by common source and common route. Further epidemiological study from the place of this patient may help in detecting the possible source for these two enterically transmitted viruses.

\section{Conclusion}

Viral hepatitis is a major public health problem in India, which is hyperendemic for HAV and HEV. Has intermediate $\mathrm{HBV}$ and $\mathrm{HCV}$ endemicity. The patients showed positivity to antibodies to HAV, HCV, HEV and HBsAg. And some coinfections were also seen. The diagnosis of these viruses becomes very important due to their prevalence rate and ill effects caused by them to the patients and carriers and also to limit the spread of this contagious disease. This is particularly important in the routine testing of potential blood donors. 


\section{References}

1. Khan WI, Sultana R, Rahman M, Akhter H, Haq JA, Ali L, Mohsin MA, Khan AK "Viral hepatitis: recent experiences from serological studies in Bangladesh". Asian Pac J Allergy Immunol 2000;18(2):99-103.

2. Pondé RAA. "The serological markers of acute infection with hepatitis A, B, C, D, E and G viruses revisited". Arch Virol 2017;162(12):3587-602.

3. Prescot, L.M Harley J.P and Klein D.A. Microbiology, International edition 4th WCB Mc.Graw-Hill. 1999.

4. Acharya SK, Madan K, Dattagupta S, Panda SK. "Viral hepatitis in India". Natl Med J India. 2006 ;19(4):203-17.

5. Locarnini S. A, Gust I. D, Ferris A. A, Stott A. C, wong M. L. "A prospective study of acute viral hepatitis with particular reference to hepatitis A". World Health Organ 1976;54:3509-199.

6. Jay H. Hoofnagle, Antonio Ponzetto, Lars R. Mathiesen, Jeanne G. Waggoner, Z. Buskell Bales and Leonard B. Seeff. "Serological diagnosis of acute viral hepatitis". Digestive Diseases and Sciences 1985;30:0163-2116.

7. Chau TN, Lai ST, Lai JY, Yuen H. "Acute viral hepatitis in Hong Kong”. Hong Kong Med J 1997;3:261-6.

8. Tondon B.N, Gandhi B.M, joshi Y.K. "Etiological spectrum of viral hepatitis and the prevalence of serological markers of hepatitis A and B virus infection in north India". Bulletin World Health Organ 1984;62(1):69-73.

9. Mishra B, Srinivasa H, Muralidharan S, Charles S, Macaden RS. "A hospital based study of hepatitis E by serology”. Ind J Pathol Microbiol 2000;43:433-36.

10. Nandi B, Hadimani P, Arunachalam R, Ganjoo RK. "Spectrum of Acute Viral Hepatitis in Southern India". Med J Armed Forces India 2009;65(1):7-9.

11. kenneth C. Hyams. "Risk of chronicity following acute hepatitis B virus infection". Clinical Infectious Dis 1995;20:992-1000. 